\title{
The Impact of HIV Genetic Polymorphisms and Subtype Differences on the Occurrence of Resistance to Antiretroviral Drugs
}

\author{
Mark A. Wainberg and Bluma G. Brenner \\ Jewish General Hospital AIDS Centre, McGill University, 3755 Cote-Ste-Catherine Road, Montreal, QC, Canada H3T 1E2 \\ Correspondence should be addressed to Mark A. Wainberg, mark.wainberg@mcgill.ca
}

Received 28 February 2012; Accepted 12 April 2012

Academic Editor: Gilda Tachedjian

Copyright (C) 2012 M. A. Wainberg and B. G. Brenner. This is an open access article distributed under the Creative Commons Attribution License, which permits unrestricted use, distribution, and reproduction in any medium, provided the original work is properly cited.

\begin{abstract}
The vast majority of reports on drug resistance deal with subtype B infections in developed countries, and this is largely due to historical delays in access to antiretroviral therapy (ART) on a worldwide basis. This notwithstanding the concept that naturally occurring polymorphisms among different non-B subtypes can affect HIV-1 susceptibility to antiretroviral drugs (ARVs) is supported by both enzymatic and virological data. These findings suggest that such polymorphisms can affect both the magnitude of resistance conferred by some major mutations as well as the propensity to acquire certain resistance mutations, even though such differences are sometimes difficult to demonstrate in phenotypic assays. It is mandatory that tools are optimized to assure accurate measurements of drug susceptibility in non-B subtypes and to recognize that each subtype may have a distinct resistance profile and that differences in resistance pathways may also impact on cross-resistance and the choice of regimens to be used in second-line therapy. Although responsiveness to first-line therapy should not theoretically be affected by considerations of viral subtype and drug resistance, well-designed long-term longitudinal studies involving patients infected by viruses of different subtypes should be carried out.
\end{abstract}

\section{Introduction}

Nonsubtype B infections are responsible for most HIV cases worldwide [1]. HIV-1 group M has been classified into subtypes, circulating and unique recombinant forms (CRF and URF, resp.), due to its significant natural genetic variation; this includes subtypes $\mathrm{A}-\mathrm{D}, \mathrm{F}-\mathrm{H}$, and $\mathrm{J}-\mathrm{K}$ and many CRFs and URFs. Although subtype $B$ is the most prevalent in the Western World (Western Europe, the Americas, Japan, and Australia), non-B subtypes predominate in the rest of the world: that is, subtype $C$ in sub-Saharan Africa, India, and Brazil, CRF01_AE in South East Asia, CRF02_AG in West Africa, and subtype A in Eastern Europe and Northern Asia [1-3]. The proportion of non-B subtypes in North and South America and Western Europe is increasing [47]. Combination antiretroviral therapy (ART) is now used in many areas of the world, and HIV resistance to antiretroviral drugs (ARVs) has widely emerged. Thus, non-B subtypes will presumably become even more common in western countries.

Reduced sensitivity to ARVs in non-B subtypes has been less well studied than in subtype $B$, mainly because of the predominance of subtype B in those countries in which ARVs first became available, coupled with the availability of genotypic and phenotypic antiretroviral drug resistance testing in such countries [8]. This notwithstanding there is a potential for genetic differences among subtypes to yield differential patterns of resistance-conferring mutations in response to ARVs and this possibility is supported by the fact that HIV-1 naturally varies in genetic content by as much as $35 \%$ among subtypes. Indeed, variation is higher in some areas of the genome $(40 \%$ in the env gene) and lower in others (8-10\% in the pol, gag, and IN genes) [8]. Since differences in codon sequences at positions associated with drug resistance mutations might predispose viral isolates from different subtypes to encode different 
amino acid substitutions, it is possible that HIV-1 genetic diversity may influence the types of resistance mutations that might eventually emerge upon drug exposure as well as the rate of emergence of such mutations and phenotypic resistance $[8,9]$. Such diversity may also affect the degree of cross-resistance to ARVs of the same class, with the potential to impact on virologic failure, clinical outcomes, and preservation of immunological responsiveness [8].

For example, studies of single dose nevirapine (sdNVP) for prevention of mother-to-child transmission (PMTCT) showed a disparity in overall resistance among subtypes, with frequencies of $69,36,19$, and $21 \%$ against NVP in women with subtypes C, D, A, and CRF02_AG infections, respectively. Often, this result occurred prior to treatment and despite the absence of resistance mutations [10-13]. Very sensitive PCR detection procedures, which reveal resistance due to minority species, have revealed a higher incidence of NVP resistance (K103N, Y181C) in 70-87\% of individuals with subtype C compared with $42 \%$ of individuals with subtype A [14-16].

Evaluations of virological and biochemical data also suggest that natural amino acid background can affect the magnitude of resistance conferred by many mutations responsible for antiretroviral drug resistance [17], as is best illustrated by HIV-2 and group $\mathrm{O}$ viruses that show highlevel innate resistance to nonnucleoside reverse transcriptase inhibitors (NNRTIs) through the presence of natural polymorphisms that can confer drug resistance (Table 1) [18, 19]. However, many studies on antiretroviral drug resistance in non-B subtypes exposed to chronic suppressive therapy have yielded less definitive results with respect to the importance of natural HIV-1 diversity as a factor leading to differences in types of drug resistance mutations and the propensity to develop drug resistance in the first place $[8,17]$.

Although genotypic ARV resistance testing is of proven benefit in deciding on best choice of ARVs for individual treatment and serves as a repository of information on $\mathrm{HIV}$ resistance mutations, several factors underscore the difficulties in defining intersubtype differences. For example, genotyping can classify the major viral subtypes, but significant proportions $(\sim 15 \%)$ of infections remain unassigned or differentially assigned using different subtyping algorithms $[8,20,21]$. Certainly, HIV resistance databases make efforts to incorporate newer subtype data into pools of data, but the availability of HIV genotypes from areas of the world with non-B subtype predominance is still comparatively low [22]. The factors responsible include lesser availability of ARV therapy, the high cost of drug resistance testing, and limited opportunities for research in resource-limited areas. In some cases, resistance tests may often be performed only on participants enrolled in study cohorts or trials but not in general practice.

\section{Resistance to Nucleoside Reverse Transcriptase Inhibitors (NRTIs)}

As an example of disparity, subtype $\mathrm{C}$ patients in Botswana treated with ZDV/ddI developed an atypical thymidine ana- logue mutation (TAM) resistance pathway $(67 \mathrm{~N} / 70 \mathrm{R} / 215 \mathrm{Y})$ compared to subtype B (the TAM 1 and TAM 2 pathways) [23]. This distinction was not observed in patients with subtype C in Malawi, India, or South Africa [24-27]. Results from Botswana also reported a high incidence of K65R $(30 \%)$ in subtype $\mathrm{C}$ patients who received $\mathrm{d} 4 \mathrm{~T} / \mathrm{ddI}$ plus NVP or efavirenz (EFV) [28]. A much larger study from Malawi detected $\mathrm{K} 65 \mathrm{R}$ or $\mathrm{K} 70 \mathrm{E}$ in $23 \%$ of patients failing first-line therapy with $\mathrm{d} 4 \mathrm{~T} / 3 \mathrm{TC} / \mathrm{NVP}$ [26], while K65R was detected in $7 \%$ and $15 \%$ of patients in South Africa failing first- or second-line regimens, respectively, whose nucleoside backbones included d4T/3TC or ddI/ZDV [29, 30]. A study from Israel also reported a high frequency of K65R in subtype C viruses from Ethiopian immigrants [31], and a report from India showed that K65R was present in about $10-12 \%$ of patients who had received $\mathrm{d} 4 \mathrm{~T} / 3 \mathrm{TC} / \mathrm{NVP}$ in first-line therapy [32]. Such differences in K65R and thymidine analogue mutations (TAMs) might be attributed to treatment regimen and disease stage [24-27].

Access to viral load testing lead in India was also associated with early detection of NRTI-treatment failure, leading to use of new, second-line regimens and preventing acquisition of TAMs and K65R [24]. Additional studies support regional differences among subtype $\mathrm{C}$ subepidemics from Ethiopia, Brazil, and sub-Saharan Africa, that impact on NRTI resistance rates as a result of different NRTI-based regimens $[8,33,34]$.

Higher rates of the K65R mutation in subtype C [26, 28, 29 ] suggest that these viruses may have a particular predisposition toward acquiring this mutation [35]. A subtype C RNA template mechanism has been proposed to explain this phenomenon that involves higher rates of K65R mutagenesis in subtype $C$ viruses than in other subtypes (Figure 1) [36, 37], and this mechanism seems to be template dependent and is independent of the source of the reverse transcriptase (RT) employed [36]. Subtype C viruses apparently have an intrinsic difficulty in synthesizing stretches of adenine homopolymeric runs that leads to template pausing at codon 65 , facilitating the acquisition of K65R under selective drug pressure $[37,38]$, whereas the subtype B template favors pausing at codon 67 that may facilitate the generation of D67N and TAMs rather than K65R [37-39]. In addition, the introduction of codons from positions 64 and 65 in the RT of subtype $\mathrm{C}$ into a subtype $\mathrm{B}$ backbone was sufficient to lead to selection of K65R by multiple NRTIs [37-39]. Figure 1 provides a pictorial representation of the preferential development of K65R in subtype C viruses.

Ultrasensitive pyrosequencing has also been used to detect the spread of K65R as transmitted and/or minority species in treatment-naïve populations [40, 41]. Patients harboring subtype $\mathrm{C}$ infections showed a higher frequency of K65R than subtype B variants (1.04\% versus $0.25 \%)$ by this method but these differences were not duplicated using limiting dilution clonal sequencing approaches [40]. While these findings are consistent with PCR-induced pausing, leading to low-level spontaneous generation of K65R in subtype C, they do not negate the higher rates of development of K65R in subtype $\mathrm{C}$ populations failing regimens containing $\mathrm{d} 4 \mathrm{~T}$, ddI, or tenofovir (TDV) [32]. The occurrence of K65R in 


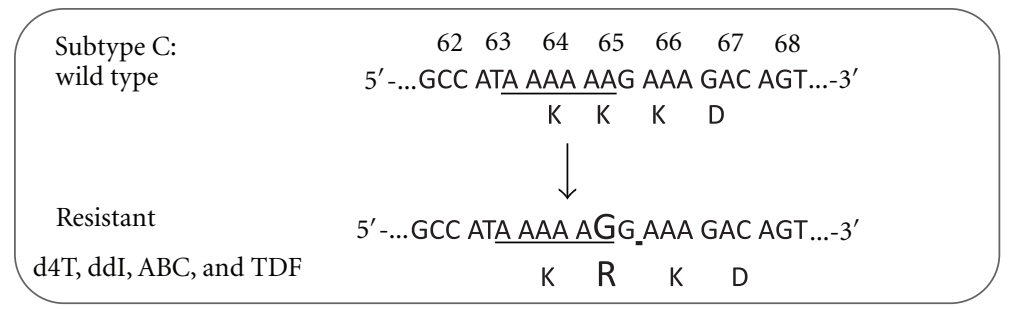

(a)

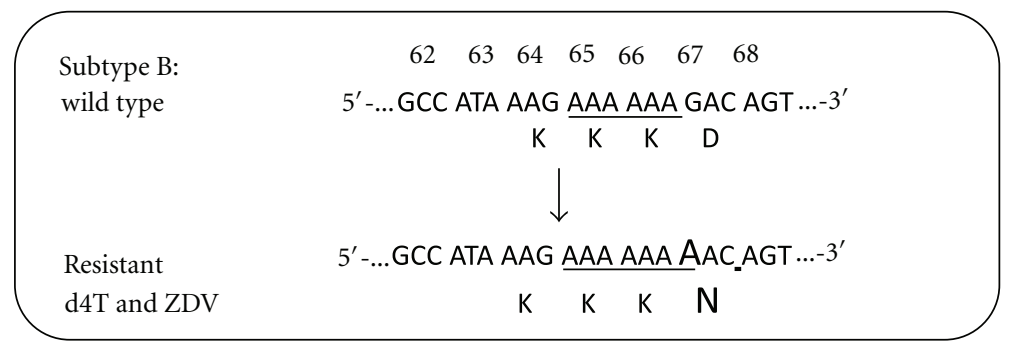

(b)

Figure 1: Subtype-specific poly-A nucleotide motifs lead to template pausing under pressure with thymidine analogues that favor K65R selection in subtype C and D67N selection in subtype B. Depiction of the template-based propensity of subtype C versus B viruses to develop the K65R mutation that is associated with broad cross-resistance among multiple members of the NRTI family of drugs. The codons located at positions 63, 64, and 65 in subtype C RT seem to be critically involved in the preferential development of K65R in subtype C. d4T: stavudine, ddI: didanosine, ABC: abacavir, TDF: tenofovir. It should be noted that the use of stavudine in particular has been shown to yeild K65R in subtype C infections with high frequency. Regimens that are based on the use of TDF and ABC, among other drugs, can help mitigate the development of the K65R mutation.

subtype $\mathrm{C}$ and CRF01_AE is also associated with the Y181C NVP mutation within the viral backbone [30, 42].

Subtype C selected the K65R mutation in drug resistance selection studies faster than subtype B under TFV pressure [35]. However, K65R may be less frequent in subtype A than other subtypes [43]. And a higher propensity to acquire TAMs was reported in patients carrying CRF_06 (AGK recombinants) as compared to patients carrying CRF02_AG from Burkina Faso [44].

The differential selection of K65R pathways in subtype $\mathrm{C}$ seems related to template differences, ddI and $\mathrm{d} 4 \mathrm{~T}$ containing regimens, as well as to the presence of Y181C. Further genotypic studies will be required to ascertain subtype differences in acquisition of resistance to NRTIs.

\section{Resistance to Nonnucleoside Reverse Transcriptase Inhibitors (NNRTIs)}

Selection studies in culture have shown that a V106M mutation commonly develops in subtype $\mathrm{C}$ viruses following drug pressure with NVP or EFV, whereas a V106A mutation is more commonly selected in subtype B. This difference is due to a nucleotide polymorphism at codon 106 in RT $[45,46]$, and the clinical importance of V106M in non-B subtypes has been confirmed in multiple studies showing that V106M is frequently seen in non-B subtypes ( $\mathrm{C}$ and CRF01_AE) after therapy with NVP or EFV $[23,25,27,47-$ $50]$.
The G190A substitution was also relatively more frequent among subtype $\mathrm{C}$ infected patients failing NNRTI-based therapy in Israel and India, and G190A/S was seen in the Israeli study as a natural polymorphism in subtype $\mathrm{C}$ from Ethiopian immigrants $[25,49]$. The frequencies of these mutations among treated patients in both studies were higher than in subtype $\mathrm{B}$ and $\mathrm{C}$ drug-naïve individuals.

Although the overall prevalence of V106M in subtype $\mathrm{C}$ is higher than in subtype B $(12 \%$ versus, $0 \%)$ in individuals failing NNRTI-based regimens, K103N (29\% versus $40 \%)$ and Y181C (12\% versus 23\%) remain important pathways for both subtype $\mathrm{C}$ and $\mathrm{B}$, respectively (http://hivdb.stanford.edu/). Only minor differences in HIV resistance pathways seem to occur among subtypes $\mathrm{A}, \mathrm{B}$, and $\mathrm{C}$ with the second generation NNRTI etravirine (ETR) [50].

\section{PR Mutations}

The results of work with protease inhibitors PIs indicate that the D30N mutation was not observed in CRF02_AG and CRF02_AE isolates in patients failing nelfinavir (NFV) therapy but rather that the N88S mutation emerged after NFV use in CRF01_AE and after indinavir [51] use in subtype B $[52,53]$. Although another study reported an absence of the D30N mutation in CRF01_AE, no information on the specific type of PIs received by the patients was available [54]. A lower frequency of $\mathrm{D} 30 \mathrm{~N}$ was seen in subtype $\mathrm{C}$ isolates from Ethiopian immigrants to Israel after NFV usage than in subtype $C$ viruses from Botswana $[55,56]$, suggesting that subtype $\mathrm{C}$ viruses from Ethiopia (the origin of the samples 
TABLE 1: Examples of polymorphisms and mutations in reverse transcriptase (RT), protease (PR), and integrase (IN) of different subtypes that may impact on emergent resistance to nucleoside and nonnucleoside reverse transcriptase inhibitors (NRTIs and NNRTIs), protease inhibitors (PIs), and integrase strand transfer inhibitors (INSTIs).

\begin{tabular}{|c|c|c|c|c|c|}
\hline Drug class & Type/group/subtype & $\begin{array}{l}\text { Polymorphism or mutation } \\
\text { associated with drug resistance }\end{array}$ & $\operatorname{Drug}(\mathrm{s})$ affected & $\begin{array}{l}\text { Mutation(s) and their } \\
\text { consequences }\end{array}$ & Reference \\
\hline \multicolumn{6}{|c|}{ Reverse transcriptase } \\
\hline NRTI & $\mathrm{C}$ & 64-65-66 KKK motif & ddI, d4T, TDF & K65R & {$[30]$} \\
\hline NRTI & HIV-2 & $\begin{array}{l}\text { T69N, V75I, V118I, L210N, T215S, } \\
\text { K219N }\end{array}$ & NRTIs & TAMs/K65R & {$[66]$} \\
\hline NNRTI & $\mathrm{C}$ & V106V & EFV, NVP & V106M & {$[45]$} \\
\hline NNRTI & G & A98S & NNRTIs & & [66] \\
\hline NNRTI & HIV-2 & $\begin{array}{l}\text { Y181I,Y188L, G190A K101A, } \\
\text { V106I, V179I }\end{array}$ & All NNRTIs & $\begin{array}{l}\text { Cross- NNRTI } \\
\text { resistance }\end{array}$ & {$[68]$} \\
\hline NNRTI & $\mathrm{O}$ & Y181C, A98S, K103R, V179E & All NNRTIs & $\begin{array}{l}\text { Cross- NNRTI } \\
\text { resistance }\end{array}$ & {$[18]$} \\
\hline \multicolumn{6}{|c|}{ Protease } \\
\hline PI & Non-B & M36I & PIs & & [59] \\
\hline PI & $\mathrm{G}, \mathrm{AE}$ & K20I & PIs & & [63] \\
\hline PI & $\mathrm{G}$ & V82I & PIs & $\mathrm{I} 82 \mathrm{M} / \mathrm{T} / \mathrm{S}$ & {$[63]$} \\
\hline PI & $\mathrm{A}, \mathrm{C}, \mathrm{F}, \mathrm{G}, \mathrm{AE}, \mathrm{AG}$ & $\mathrm{L} 89 \mathrm{M}$ & PIs & L89I & [71] \\
\hline PI & HIV-2 & $\begin{array}{c}\text { L10I/V, K 20V, V32I, M36I, M46I, } \\
\text { I47V, L63E/K, A71V, G73A, V77T, } \\
\text { V82I/L, }\end{array}$ & PIs & APV and other PIs & {$[68]$} \\
\hline \multicolumn{6}{|c|}{$\begin{array}{ll}\text { Integrase } \\
\end{array}$} \\
\hline \multirow{2}{*}{ INSTIs } & B & R263 & MK-2048, DTG & R263K & {$[85]$} \\
\hline & $\mathrm{C}$ & G118 & MK-2048, DTG & G118R & {$[82]$} \\
\hline
\end{tabular}

ddI: didanosine; d4T: stavudine; TFV, tenofovir: EFV, efavirenz: NVP, nevirapine: DTG, dolutegravir.

identified in Israel) and southern Africa might behave in different fashion. M89I/V mutations were observed in F, G, and C subtypes but not in other subtypes [26], and the V82I natural polymorphism in subtype $\mathrm{G}$ led to the emergence of I82M/T/S in treatment failure [57]. The L90M mutation is rare in subtype $\mathrm{F}$ but common in subtype B from Brazil [58], and a recent paper suggests that polymorphisms at position 36 in PR may be important in determining the emergence of specific patterns of resistance mutations among viruses of different subtypes [59].

To gain an understanding of the underlying mechanisms leading to the overall higher preponderance of D30N in subtype B relative to other subtypes, molecular dynamic simulations were performed. D30N appeared to selectively confer resistance to NFV in subtype B by increasing the flexibility of the protease (PR) flap region and destabilizing the PR inhibitor complex [60]. In subtype C, D30N required the accessory $\mathrm{N} 83 \mathrm{~T}$ mutation to confer resistance and rescue fitness [61].

Two comprehensive surveys reported differences in natural protease polymorphisms among non-B subtypes $[62,63]$ and positions less frequently mutated in non- $B$ subtypes than in subtype $B$ after exposure to ARVs. Residues of importance in subtype A in PR were at positions 10,20, and 63, whereas, in subtype C, they were at residues $20,53,63,74$, and 82 . Other differences were at residues 13 and 20 in subtype D, residues $10,14,20$, and 77 in subtype $F$, residues 20,67 ,
73,82 , and 88 in subtype $G$, residues $20,63,82$, and 89 in CRF01_AE, and residue 20 in CRF02_AG [63].

Higher rates of accumulation of NRTI and PI resistance mutations and equal rates of emergence of NNRTI mutations were also found in subtype B compared to C [64]. A study from southern Brazil also showed a lower frequency of primary resistance to PIs in subtype $\mathrm{C}$ compared to subtype $\mathrm{B}$, suggesting that PI mutations may be less well tolerated at the structural level in subtype C [65].

However, HIV-1 subtype diversity has not limited the overall benefit of ART (Table 1). This notwithstanding there are subtype differences in the type and preference of pathways of resistance with some mutations emerging almost exclusively in some non-B subtypes, for example, the protease mutation $82 \mathrm{M}$ in subtype $\mathrm{G}$ versus $82 \mathrm{~A} / \mathrm{F} / \mathrm{S}$ in the others, $88 \mathrm{D}$ in subtype $\mathrm{B}$ versus $88 \mathrm{~S}$ in subtypes $\mathrm{C}$ and CRF02_AG [66]. Furthermore, HIV-2 has major mutations in regard to NRTIs, NNRTIs, and PIs, which contribute to innate NNRTI resistance and rapid development of multiclass drug resistance (Table 1) [67, 68]. The V106M RT mutation in subtypes C and A versus V106A in subtype $\mathrm{B}$ is observed with resistance against NVP and EFV. Polymorphisms at RT residue 98, common in subtype G, are associated with NNRTI resistance in subtype B and may lower the resistance barrier and duration of efficacy of some NNRTIs [69]. The frequency of some resistance mutations shared by $\mathrm{B}$ and non-B subtypes can vary after failure of 
first-line therapeutic regimens, as in the case of the K65R mutation. Differences in type and frequency of resistance mutations should not be underestimated. However, the TAM pathway $67 \mathrm{~N} / 70 \mathrm{R} / 215 \mathrm{Y}$ found in subtype $\mathrm{C}$ in Botswana will probably be adequately detected by most resistance algorithms, since it does not involve new mutations.

A lower risk for accumulation of major (primary) resistance mutations in subtype $\mathrm{C}$ than $\mathrm{B}$ has been reported [64]. The major mutations that emerged in both subtypes were the same. Since both subtypes B and C patients had similar profiles of virological failure after use of the same ART regimens, this rules out ancillary factors responsible for these differences. Minor mutations in subtype B PR may appear as frequent natural polymorphisms in several nonB subtypes (e.g., M36I, L89M) $[58,59]$. The fact that the L89M polymorphism can lead to the M89I mutation that confers resistance to PIs suggests that there might be a lower accumulation of major mutations in $\mathrm{C}$ subtypes, if natural polymorphisms act similarly in subtype $\mathrm{C}$ as they do when present as secondary resistance mutations in subtype $\mathrm{B}$.

The majority of non-B HIV-1 subtype isolates possess wild-type susceptibilities similar to those of subtype B wild-type isolates. Compared to B subtypes, diminished susceptibilities among wild-type isolates have been found for CRF02_AG recombinant viruses in three different studies in regard to ATV and NFV $[63,69,70]$. No study has yet assigned statistical significance of drug susceptibility levels due to polymorphisms and small sample size. One analysis performed molecular modeling and suggested that distortions in the $\mathrm{K} 26$ pocket of $\mathrm{A} / \mathrm{G}$ proteases appear to be responsible for a lower binding energy of NFV and hence lower susceptibility of $\mathrm{A} / \mathrm{G}$ viruses to this drug [70]. A/G isolates with lower susceptibilities to certain PIs (NFV and atazanavir (ATV)) have also been found. One study has detected an important proportion of WT isolates with lower susceptibilities to ATV [71]. In most cases, phenotypes have been determined by commercial or in-house assays that were developed primarily to measure B-subtype drug susceptibilities based on the laboratory adapted strains NL43 or HXB2, through use of a modified clone of a laboratory strain that lacks both the terminal part of Gag and most of Pol. It should be recognized that most commercial assays do not monitor polymorphisms, and indeed sequences that lie within particular regions, such as the substrates of PR within gag or the RNaseH and connection domains within pol, can influence drug resistance in both $B$ and non-B subtypes but may not be easily recognized. Although some work has been carried out in this field, it is clear that other studies are required [72-75].

There are few data on the potential for cross-resistance to PIs among non-B subtypes in regard to NFV, although there is a tendency to select for the L90M pathway instead of D30N in subtype C. Competition fitness assays support the notion that subtype $\mathrm{C}$ viruses bearing $\mathrm{D} 30 \mathrm{~N}$ are impaired in replicative fitness, a finding that may explain the above results [61].

Thermodynamic studies performed on target-inhibitor interactions in PR have specifically described a lower affinity of non-B subtype proteases for PIs and amplification of primary resistance mutations on the basis of polymorphisms that are present in background.

In addition to the foregoing, interesting results on polymorphisms that confer hypersusceptibility to some PIs have been recently reported [76]. Some of these polymorphisms can potentially delay acquisition of drug resistance and may therefore enhance the long-term effectiveness of relevant drugs.

\section{Integrase Inhibitors and Drug Resistance}

New data are emerging that subtype differences are also present in regard to integrase strand transfer inhibitors (INSTIs) despite the fact that HIV-1 subtype B and C wildtype integrase (IN) enzymes are similarly susceptible to clinically approved INSTIs [77-81]. This notwithstanding there are now data to indicate that the presence of resistance mutations may differentially affect susceptibility to specific INSTIs in viruses of different subtypes [77]. Moreover, such data have been obtained both in tissue culture using recombinant viruses of different subtypes that contain specific IN mutations as well as in biochemical integrase strand transfer and integrase 3'synthetase assays, in which specific drug resistance mutations have been introduced into recombinant purified integrase enzymes derived from either subtype $B$ or subtype $C$ viruses [77].

Of particular interest may be that a novel next-generation INSTI termed MK-2078 with a higher genetic barrier for selection of resistance than either raltegravir (RAL) or elvitegravir (EGV) was able to differentially select for a novel G118R substitution in IN in subtype C compared with subtype B viruses [82]. This mutation conferred only slight resistance to MK-2048 but gave rise to 25 -fold resistance against RAL when it was present together with a polymorphic substitution at position L74M in CRF02-AG cloned patient isolates [83]. It is also well known that INSTI Q148RHK resistance mutations that affect susceptibility to a novel INSTI, dolutegravir (DTG) in HIV-1 subtype B may not affect susceptibility of subtype $\mathrm{C}$ viruses or HIV-2 viruses and IN enzymes to the latter compound [84].

Finally, tissue culture selection with DTG has identified a novel R263K resistance mutation in subtype B but not subtype $C$ viruses [85]. In contrast, the same series of selections with DTG in subtype $C$ viruses yielded the same G118R mutation that had previously been obtained with MK-2048, also in subtype C. This raises the possibility that G118R may have the potential to be an important resistance mutation for next-generation INSTIs in subtype $\mathrm{C}$ viruses but that this role may be played by $\mathrm{R} 263 \mathrm{~K}$ in the context of subtype B viruses. Of course, definitive information on this topic may have to await the widespread clinical use of DTG and the characterization of mutations within IN that may arise in the event of rising viral loads and treatment failure.

\section{Clinical Practice}

HIV resistance in non-B subtypes has rarely been reported on the basis of single drugs or NRTI backbones but, rather, mutations have been reported for specific drug classes. 
Cross-resistance can be estimated only for some NRTIs and NNRTIs but not for most PIs that are the only drugs eligible as part of second-line regimens in most regions of the world. The potential for cross-resistance to NFV in viruses of CRF01_AE and CRF02_AG origin could be higher than has been observed in subtype $B$, due to the preferential selection of the N88S and L90M substitutions, although such data are not yet available for most PIs in the context of non-subtype $B$ viruses. NRTI backbones may also vary in the mutation profile they select for according to drug combinations that are used. Newer compounds (e.g., TFV and ATV/r) are now preferred both in resource-rich countries and non-B subtype prevalent areas. Although HIV resistance databases continue to enter HIV genotype data from nonB subtype variants, few data sets are available to date (stanford HIV resistance database, Agence Nationale pour la Recherche sur le SIDAFrance (ANRS), etc.) for drugs that have become part of first-line therapy in developed countries, for example, TDF, ATV, darunavir, ETR, and RAL. In this context as well, it is relevant that some studies have attempted to address the clinical impact of HIV diversity on treatment response as well as the limitations of such approaches $[86,87]$.

\section{Future Considerations}

The preferential emergence of some mutations and changes in the frequency of these mutations in select non-B subtypes needs greater attention and research on the role of polymorphisms in nonsubtype $B$ viruses that increase in frequency after drug exposure and that may contribute to drug resistance (e.g., A98G/S in RT and M36I and K20I in PR) [88] should be priorized, particularly in parts of Africa in which treatment failure has been reported in as many as $40 \%$ of patients after two years [89] and in India where resistance rates of $80 \%$ to two drug classes have been reported after failure of first-line regimens that employed various NRTI/NNRTI combinations [90]. To date, no study has tested the degree of resistance or cross-resistance that certain mutational combinations $(67 \mathrm{~N} / 70 \mathrm{R} / 215 \mathrm{Y})$ may confer in tissue culture. Newer studies should assess pre- and posttreatment genotypes in order to determine associations of certain polymorphisms with drug resistance, including variations of polymorphisms in variants of the same subtype that are located in different geographical regions. This would improve the appropriateness of use of certain drugs over others in the context of second- or third-line therapeutic regimens.

The different studies conducted in populations affected by nonsubtype $\mathrm{B}$ viruses are too heterogeneous to permit pooling of data [8]. Such studies have addressed different research questions and used nonequivalent NRTI backbones (e.g., ZVD/ddI and ZDV/3TC) and have also grouped mutations by drug class without providing information on the nature of the regimen at virologic failure. Resistance has also been reported in different ways (e.g., different algorithms or resistance lists), making it difficult to relate resistance mutations to a specific drug or combination of drugs. More longitudinal studies on response to first-line ARV combinations are needed to better recognize intersubtype differences. Pre- and posttherapy genotype resistance testing is also desirable.

\section{Conclusions}

Virological and biochemical data provide compelling evidence on the differential effect of genetic background on both the type and degree of HIV-1 antiretroviral drug resistance. Genetic background can affect the degree of protein binding caused by primary mutations and restore the function of PR to a differential degree in different subtypes based on background polymorphisms, although this effect was not discernible in the absence of typical major resistance mutations but rather when particular backgrounds of combinations of major resistance mutations and background polymorphisms were represented. Clearly, some background polymorphisms can act as secondary resistance substitutions.

Phenotypic assays have failed to find differences of large magnitude in the susceptibilities of HIV B versus non-B subtypes, consistent with what has been learned at a molecular level. Unfortunately, only few datasets exist on relative susceptibility levels among subtypes carrying specific major resistance mutations, and more information is required, particularly because many polymorphisms in non$B$ viruses are considered to be secondary resistance mutations since they can emerge after drug exposure in subtype B viruses. The effect of such polymorphisms within different genetic backgrounds cannot always be extrapolated to non-B subtypes and might sometimes contribute to higher levels of resistance depending on genetic backbone. They could also have either a neutral effect or hypersensitize HIV to ARVs, and I93L is an example of a secondary resistance mutation in subtype B that in subtype C causes hypersusceptibility to PIs [61].

Novel NNRTI resistance mutations in subtype $C$ were not recognized in subtype $B$. In tissue culture, subtype $C$ can acquire a V106M mutation under NNRTI drug pressure compared to V106A in subtype B. V106M can confer broad cross-resistance to an extent that supersedes that conferred by V106A.

The acquisition of resistance could have important implications in regard to durability of therapy. In culture, the emergence of the K65R mutation is quicker in subtype $\mathrm{C}$ than in B $[30,35]$, and several biochemical mechanisms have been proposed to explain this observation, based on subtype C templates [36-38, 91]. K65R has been seen in approximately $70 \%$ of patients failing ddI-containing nucleoside backbones in Botswana [28] but does not appear to emerge frequently in subtype $\mathrm{C}$ patients who have received either TDF or TDF/FTC as part of triple therapy [30], a possible reflection of the use of well-tolerated effective drugs that have long mutually reinforcing intracellular halflives that act in combination to suppress viral replication and prevent the emergence of resistance mutations. Higher numbers of patients and longer followup will be required to determine if there is a consistent impact of subtype $\mathrm{C}$ in the emergence of K65R in the clinic.

Multiple in vitro and clinical studies have confirmed that PR and Gag can act as a functional unit and coevolve 
when HIV is subject to drug pressure. Both genes can clearly mutate under PI pressure, and Gag mutations can act as compensatory substitutions that may increase levels of viral replication capacity and resistance. The recombinant phenotyping systems used for clinical samples do not now adequately monitor Gag. While differences among Gag may vary between only -2 to 2.5 -fold between subtypes, different subtypes might develop compensatory Gag mutations at different rates, establishing a need to take Gag into account in determining a phenotype. One study reported that a recombinant construct included Gag of clinical origin but did not test the same subtypes as were reported in other work [57].

Although various mutations can impact on drug sensitivity to differential extent, such information cannot yet be generated with regard to non-B subtypes due to a paucity of paired phenotypic and genotypic data. Three studies analyzed genotypes and phenotypes of non-B subtypes in clinical trials: one on use of single dose NVP for prevention of mother-to-child transmission and two on double and triple NRTI combinations that are no longer used [8].

Cross-resistance acquires importance in settings with limited access to antiretroviral therapy, and few in vitro comparative data are available for PIs in non-B subtypes. However, such data may be crucial to understanding crossresistance to specific drugs $[58,59]$, since some PIs may be the only potentially accessible option for drug sequencing in salvage therapy in many resource-limited settings. The fact that resistance to PIs commonly requires that large numbers of resistance mutations be present may yield a situation in which the individual contribution of any single mutation to drug resistance, with some exceptions, will be limited, a definite advantage of using drugs with a high genetic barrier toward the development of drug resistance. Thus, differences among subtypes with regard to development of drug resistance are more likely to be important for NRTIs and NNRTIs than for PIs. Clearly, large numbers of paired samples need to be systematically collected from naïve and treated patients infected with subtypes $\mathrm{C}, \mathrm{AE}$, $A G, A$, and $G$, in order for genotypic and phenotypic analysis to be conducted for both established drug classes as well as for newer classes of drugs such as inhibitors of integrase.

Finally, this paper has focused on classes of HIV drugs for which significant datasets are available in regard to subtypes and differential drug resistance. Limitations of both space and available datasets have precluded us from discussing the topic of entry inhibitors. However, most available data suggest that the only two approved entry inhibitors, that is, the fusion inhibitor, enfuvirtide, and the CCR5 entry antagonist, maraviroc, are both active against HIV isolates of multiple subtypes.

\section{Acknowledgments}

Work in our lab is supported by the Canadian Institutes of Health Research and by the Canadian Foundation for AIDS Research.

\section{References}

[1] K. K. Ariën, G. Vanham, and E. J. Arts, "Is HIV-1 evolving to a less virulent form in humans?" Nature Reviews Microbiology, vol. 5, no. 2, pp. 141-151, 2007.

[2] E. A. J. M. Soares, R. P. Santos, J. A. Pellegrini, E. Sprinz, A. Tanuri, and M. A. Soares, "Epidemiologic and molecular characterization of human immunodeficiency virus type 1 in southern Brazil," Journal of Acquired Immune Deficiency Syndromes, vol. 34, no. 5, pp. 520-526, 2003.

[3] E. A. J. M. Soares, A. M. B. Martínez, T. M. Souza et al., "HIV1 subtype C dissemination in southern Brazil," AIDS, vol. 19, supplement 4, pp. S81-S86, 2005.

[4] C. A. Brennan, C. Brites, P. Bodelle et al., "HIV-1 strains identified in Brazilian blood donors: significant prevalence of B/F1 recombinants," AIDS Research and Human Retroviruses, vol. 23, no. 11, pp. 1434-1441, 2007.

[5] D. Locateli, P. H. Stoco, A. T. L. de Queiroz et al., "Molecular epidemiology of HIV-1 in Santa Catarina State confirms increases of subtype c in southern Brazil," Journal of Medical Virology, vol. 79, no. 10, pp. 1455-1463, 2007.

[6] A. Holguín, M. de Mulder, G. Yebra, M. López, and V. Soriano, "Increase of non-B subtypes and recombinants among newly diagnosed HIV-1 native spaniards and immigrants in Spain," Current HIV Research, vol. 6, no. 4, pp. 327-334, 2008.

[7] D. Descamps, M. L. Chaix, P. André et al., "French national sentinel survey of antiretroviral drug resistance in patients with HIV-1 primary infection and in antiretroviral-naive chronically infected patients in 2001-2002," Journal of Acquired Immune Deficiency Syndromes, vol. 38, no. 5, pp. 545552, 2005.

[8] B. G. Brenner, "Resistance and viral subtypes: how important are the differences and why do they occur?" Current Opinion in HIV and AIDS, vol. 2, no. 2, pp. 94-102, 2007.

[9] R. Kantor, "Impact of HIV-1 pol diversity on drug resistance and its clinical implications," Current Opinion in Infectious Diseases, vol. 19, no. 6, pp. 594-606, 2006.

[10] T. D. Toni, B. Masquelier, E. Lazaro et al., "Characterization of nevirapine (NVP) resistance mutations and HIV type 1 subtype in women from Abidjan (Cote d'Ivoire) after NVP single-dose prophylaxis of HIV type 1 mother-to-child transmission," AIDS Research and Human Retroviruses, vol. 21, no. 12, pp. 1031-1034, 2005.

[11] S. H. Eshleman, D. R. Hoover, S. Chen et al., "Nevirapine (NVP) resistance in women with HIV-1 subtype C, compared with subtypes $\mathrm{A}$ and $\mathrm{D}$, after the administration of single-dose NVP," Journal of Infectious Diseases, vol. 192, no. 1, pp. 30-36, 2005.

[12] S. H. Eshleman, J. D. Church, S. Chen et al., "Comparison of HIV-1 mother-to-child transmission after single-dose nevirapine prophylaxis among African women with subtypes A, C, and D," Journal of Acquired Immune Deficiency Syndromes, vol. 42, no. 4, pp. 518-521, 2006.

[13] M. L. Chaix, D. K. Ekouevi, F. Rouet et al., "Low risk of nevirapine resistance mutations in the prevention of mother-to-child transmission of HIV-1: Agence Nationale de Recherches sur le SIDA Ditrame Plus, Abidjan, Cote d'Ivoire," Journal of Infectious Diseases, vol. 193, no. 4, pp. 482-487, 2006.

[14] J. A. Johnson, J. F. Li, L. Morris et al., "Emergence of drugresistant HIV-1 after intrapartum administration of singledose nevirapine is substantially underestimated," Journal of Infectious Diseases, vol. 192, no. 1, pp. 16-23, 2005. 
[15] T. S. Flys, S. Chen, D. C. Jones et al., "Quantitative analysis of HIV-1 variants with the K103N resistance mutation after single-dose nevirapine in women with HIV-1 subtypes A, C, and D," Journal of Acquired Immune Deficiency Syndromes, vol. 42, no. 5, pp. 610-613, 2006.

[16] T. Flys, D. V. Nissley, C. W. Claasen et al., "Sensitive drugresistance assays reveal long-term persistence of HIV-1 variants with the K103N nevirapine (NVP) resistance mutation in some women and infants after the administration of singledose NVP: HIVNET 012," Journal of Infectious Diseases, vol. 192, no. 1, pp. 24-29, 2005.

[17] J. L. Martínez-Cajas, N. Pant-Pai, M. B. Klein, and M. A. Wainberg, "Role of genetic diversity amongst HIV-1 non-B subtypes in drug resistance: a systematic review of virologic and biochemical evidence," AIDS Reviews, vol. 10, no. 4, pp. 212-223, 2008.

[18] D. Descamps, G. Collin, F. Letourneur et al., "Susceptibility of human immunodeficiency virus type 1 group $\mathrm{O}$ isolates to antiretroviral agents: in vitro phenotypic and genotypic analyses," Journal of Virology, vol. 71, no. 11, pp. 8893-8898, 1997.

[19] E. Tuaillon, M. Gueudin, V. Lemée et al., "Phenotypic susceptibility to nonnucleoside inhibitors of virion-associated reverse transcriptase from different HIV types and groups," Journal of Acquired Immune Deficiency Syndromes, vol. 37, no. 5, pp. 1543-1549, 2004.

[20] L. Vergne, J. Snoeck, A. Aghokeng et al., "Genotypic drug resistance interpretation algorithms display high levels of discordance when applied to non-B strains from HIV-1 naive and treated patients," FEMS Immunology and Medical Microbiology, vol. 46, no. 1, pp. 53-62, 2006.

[21] R. Gifford, T. de Oliveira, A. Rambaut et al., "Assessment of automated genotyping protocols as tools for surveillance of HIV-1 genetic diversity," AIDS, vol. 20, no. 11, pp. 1521-1529, 2006.

[22] S. Y. Rhee, R. Kantor, D. A. Katzenstein et al., "HIV-1 pol mutation frequency by subtype and treatment experience: extension of the HIVseq program to seven non-B subtypes," AIDS, vol. 20, no. 5, pp. 643-651, 2006.

[23] V. Novitsky, C. W. Wester, V. DeGruttola et al., "The reverse transcriptase $67 \mathrm{~N} 70 \mathrm{R} 215 \mathrm{Y}$ genotype is the predominant TAM pathway associated with virologic failure among HIV type 1C-infected adults treated with ZDV/ddI-containing HAART in Southern Africa," AIDS Research and Human Retroviruses, vol. 23, no. 7, pp. 868-878, 2007.

[24] R. E. Barth, A. M. Wensing, H. A. Tempelman, R. Moraba, R. Schuurman, and A. I. Hoepelman, "Rapid accumulation of nonnucleoside reverse transcriptase inhibitor-associated resistance: evidence of transmitted resistance in rural South Africa," AIDS, vol. 22, no. 16, pp. 2210-2212, 2008.

[25] A. Deshpande, V. Jauvin, N. Magnin et al., "Resistance mutations in subtype C HIV type 1 isolates from Indian patients of Mumbai receiving NRTIs plus NNRTIs and experiencing a treatment failure: resistance to AR," AIDS Research and Human Retroviruses, vol. 23, no. 2, pp. 335-340, 2007.

[26] M. C. Hosseinipour, J. J. G. van Oosterhout, R. Weigel et al., "The public health approach to identify antiretroviral therapy failure: high-level nucleoside reverse transcriptase inhibitor resistance among Malawians failing first-line antiretroviral therapy," AIDS, vol. 23, no. 9, pp. 1127-1134, 2009.

[27] V. C. Marconi, H. Sunpath, Z. Lu et al., "Prevalence of HIV-1 drug resistance after failure of a first highly active antiretroviral therapy regimen in KwaZulu Natal, South Africa," Clinical Infectious Diseases, vol. 46, no. 10, pp. 1589-1597, 2008.
[28] F. Doualla-Bell, A. Avalos, B. Brenner et al., "High prevalence of the K65R mutation in human immunodeficiency virus type 1 subtype $\mathrm{C}$ isolates from infected patients in Botswana treated with didanosine-based regimens," Antimicrobial Agents and Chemotherapy, vol. 50, no. 12, pp. 4182-4185, 2006.

[29] C. Orrell, R. P. Walensky, E. Losina, J. Pitt, K. A. Freedberg, and R. Wood, "HIV type-1 clade $\mathrm{C}$ resistance genotypes in treatment-naive patients and after first virological failure in a large community antiretroviral therapy programme," Antiviral Therapy, vol. 14, no. 4, pp. 523-531, 2009.

[30] B. G. Brenner and D. Coutsinos, "The K65R mutation in HIV1 reverse transcriptase: genetic barriers, resistance profile and clinical implications," HIV Therapy, vol. 3, no. 6, pp. 583-594, 2009.

[31] D. Turner, E. Shahar, E. Katchman et al., "Prevalence of the K65R resistance reverse transcriptase mutation in different HIV-1 subtypes in Israel," Journal of Medical Virology, vol. 81, no. 9, pp. 1509-1512, 2009.

[32] A. Deshpande, A. C. Jeannot, M. H. Schrive, L. Wittkop, P. Pinson, and H. J. Fleury, "Analysis of RT sequences of subtype C HIV-type 1 isolates from indian patients at failure of a firstline treatment according to clinical and/or immunological WHO guidelines," AIDS Research and Human Retroviruses, vol. 26, no. 3, pp. 343-350, 2010.

[33] W. Ayele, Y. Mekonnen, T. Messele et al., "Differences in HIV type 1 RNA plasma load profile of closely related cocirculating ethiopian subtype C strains: C and C', AIDS Research and Human Retroviruses, vol. 26, no. 7, pp. 805-813, 2010.

[34] R. Fontella, M. A. Soares, and C. G. Schrago, "On the origin of HIV-1 subtype C in South America," AIDS, vol. 22, no. 15, pp. 2001-2011, 2008.

[35] B. G. Brenner, M. Oliveira, F. Doualla-Bell et al., "HIV-1 subtype $\mathrm{C}$ viruses rapidly develop K65R resistance to tenofovir in cell culture," AIDS, vol. 20, no. 9, pp. F9-F13, 2006.

[36] C. F. Invernizzi, D. Coutsinos, M. Oliveira, D. Moisi, B. G. Brenner, and M. A. Wainberg, "Signature nucleotide polymorphisms at positions 64 and 65 in reverse transcriptase favor the selection of the K65R resistance mutation in HIV-1 subtype C," Journal of Infectious Diseases, vol. 200, no. 8, pp. 1202-1206, 2009.

[37] D. Coutsinos, C. F. Invernizzi, H. Xu et al., "Template usage is responsible for the preferential acquisition of the $\mathrm{K} 65 \mathrm{R}$ reverse transcriptase mutation in subtype $C$ variants of human immunodeficiency virus type 1," Journal of Virology, vol. 83, no. 4, pp. 2029-2033, 2009.

[38] D. Coutsinos, C. F. Invernizzi, H. Xu, B. G. Brenner, and M. A. Wainberg, "Factors affecting template usage in the development of K65R resistance in subtype C variants of HIV type-1," Antiviral Chemistry and Chemotherapy, vol. 20, no. 3, pp. 117-131, 2010.

[39] P. R. Harrigan, C. W. Sheen, V. S. Gill et al., "Silent mutations are selected in HIV-1 reverse transcriptase and affect enzymatic efficiency," AIDS, vol. 22, no. 18, pp. 2501-2508, 2008.

[40] V. Varghese, E. Wang, F. Babrzadeh et al., "Nucleic acid template and the risk of a PCR-induced HIV-1 drug resistance mutation," PLoS ONE, vol. 5, no. 6, Article ID e10992, 2010.

[41] R. T. D’Aquila, A. M. Geretti, J. H. Horton et al., “Tenofovir (TDF)-selected or abacavir (ABC)-selected low-frequency HIV type 1 subpopulations during failure with persistent viremia as detected by ultradeep pyrosequencing," AIDS Research and Human Retroviruses, vol. 27, no. 2, pp. 201-209, 2011. 
[42] M. Zolfo, J. M. Schapiro, V. Phan et al., "Genotypic impact of prolonged detectable HIV type 1 RNA viral load after HAART failure in a CRF01-AE-infected cohort," AIDS Research and Human Retroviruses, vol. 27, no. 7, pp. 727-735, 2011.

[43] R. K. Gupta, I. L. Chrystie, S. O'Shea, J. E. Mullen, R. Kulasegaram, and C. Y. W. Tong, "K65R and Y181C are less prevalent in HAART-experienced HIV-1 subtype A patients," AIDS, vol. 19, no. 16, pp. 1916-1919, 2005.

[44] D. M. Tebit, L. Sangaré, A. Makamtse et al., "HIV drug resistance pattern among HAART-exposed patients with suboptimal virological response in Ouagadougou, Burkina Faso," Journal of Acquired Immune Deficiency Syndromes, vol. 49, no. 1, pp. 17-25, 2008.

[45] H. Loemba, B. Brenner, M. A. Parniak et al., "Genetic divergence of human immunodeficiency virus type 1 Ethiopian clade $\mathrm{C}$ reverse transcriptase (RT) and rapid development of resistance against nonnucleoside inhibitors of RT," Antimicrobial Agents and Chemotherapy, vol. 46, no. 7, pp. 2087-2094, 2002.

[46] B. Brenner, D. Turner, M. Oliveira et al., "A V106M mutation in HIV-1 clade $\mathrm{C}$ viruses exposed to efavirenz confers crossresistance to non-nucleoside reverse transcriptase inhibitors," AIDS, vol. 17, no. 1, pp. F1-F5, 2003.

[47] L. Y. Hsu, R. Subramaniam, L. Bacheler, and N. I. Paton, "Characterization of mutations in CRF01_AE virus isolates from antiretroviral treatment-naive and -experienced patients in Singapore," Journal of Acquired Immune Deficiency Syndromes, vol. 38, no. 1, pp. 5-13, 2005.

[48] L. Rajesh, R. Karunaianantham, P. R. Narayanan, and S. Swaminathan, "Antiretroviral drug-resistant mutations at baseline and at time of failure of antiretroviral therapy in HIV type 1-coinfected TB patients," AIDS Research and Human Retroviruses, vol. 25, no. 11, pp. 1179-1185, 2009.

[49] Z. Grossman, V. Istomin, D. Averbuch et al., "Genetic variation at NNRTI resistance-associated positions in patients infected with HIV-1 subtype C," AIDS, vol. 18, no. 6, pp. 909-915, 2004.

[50] M. T. Lai, M. Lu, P. J. Felock et al., "Distinct mutation pathways of non-subtype B HIV-1 during in vitro resistance selection with nonnucleoside reverse transcriptase inhibitors," Antimicrobial Agents and Chemotherapy, vol. 54, no. 11, pp. 4812-4824, 2010.

[51] G. Aad, B. Abbott, J. Abdallah et al., "Search for new phenomena in $t \bar{t}$ events with large missing transverse momentum in proton-proton collisions at $\sqrt{s}=7 \mathrm{TeV}$ with the ATLAS detector," Physical Review Letters, vol. 108, no. 4, Article ID 041805, 2012.

[52] K. Ariyoshi, M. Matsuda, H. Miura, S. Tateishi, K. Yamada, and W. Sugiura, "Patterns of point mutations associated with antiretroviral drug treatment failure in CRF01_AE (subtype E) infection differ from subtype B infection," Journal of Acquired Immune Deficiency Syndromes, vol. 33, no. 3, pp. 336-342, 2003.

[53] M. L. Chaix, F. Rouet, K. A. Kouakoussui et al., "Genotypic human immunodeficiency virus type 1 drug resistance in highly active antiretroviral therapy-treated children in Abidjan, Cote d'Ivoire," Pediatric Infectious Disease Journal, vol. 24, no. 12, pp. 1072-1076, 2005.

[54] C. Sukasem, V. Churdboonchart, W. Sukeepaisarncharoen et al., "Genotypic resistance profiles in antiretroviral-naive HIV-1 infections before and after initiation of first-line HAART: impact of polymorphism on resistance to therapy," International Journal of Antimicrobial Agents, vol. 31, no. 3, pp. 277-281, 2008.
[55] Z. Grossman, E. E. Paxinos, D. Averbuch et al., "Mutation D30N is not preferentially selected by human immunodeficiency virus type 1 subtype $\mathrm{C}$ in the development of resistance to nelfinavir," Antimicrobial Agents and Chemotherapy, vol. 48, no. 6, pp. 2159-2165, 2004.

[56] F. Doualla-Bell, A. Avalos, T. Gaolathe et al., "Impact of human immunodeficiency virus type 1 subtype $\mathrm{C}$ on drug resistance mutations in patients from Botswana failing a nelfinavir-containing regimen," Antimicrobial Agents and Chemotherapy, vol. 50, no. 6, pp. 2210-2213, 2006.

[57] A. T. Dumans, M. A. Soares, E. S. Machado et al., "Synonymous genetic polymorphisms within Brazilian human immunodefidency virus type 1 subtypes may influence mutational routes to drug resistance," Journal of Infectious Diseases, vol. 189, no. 7, pp. 1232-1238, 2004.

[58] A. Calazans, R. Brindeiro, P. Brindeiro et al., "Low accumulation of L90M in protease from subtype F HIV-1 with resistance to protease inhibitors is caused by the L89M polymorphism," Journal of Infectious Diseases, vol. 191, no. 11, pp. 1961-1970, 2005.

[59] I. Lisovsky, S. M. Schader, J. L. Martinez-Cajas, M. Oliveira, D. Moisi, and M. A. Wainberg, "HIV-1 protease codon 36 polymorphisms and differential development of resistance to nelfinavir, lopinavir, and atazanavir in different HIV-1 subtypes," Antimicrobial Agents and Chemotherapy, vol. 54, no. 7, pp. 2878-2885, 2010.

[60] R. O. Soares, P. R. Batista, M. G. S. Costa, L. E. Dardenne, P. G. Pascutti, and M. A. Soares, "Understanding the HIV-1 protease nelfinavir resistance mutation $\mathrm{D} 30 \mathrm{~N}$ in subtypes $\mathrm{B}$ and C through molecular dynamics simulations," Journal of Molecular Graphics and Modelling, vol. 29, no. 2, pp. 137-147, 2010.

[61] L. M. F. Gonzalez, R. M. Brindeiro, R. S. Aguiar et al., "Impact of nelfinavir resistance mutations on in vitro phenotype, fitness, and replication capacity of human immunodeficiency virus type 1 with subtype B and C proteases," Antimicrobial Agents and Chemotherapy, vol. 48, no. 9, pp. 3552-3555, 2004.

[62] R. Kantor and D. Katzenstein, "Polymorphism in HIV-1 nonsubtype $b$ protease and reverse transcriptase and its potential impact on drug susceptibility and drug resistance evolution," AIDS Reviews, vol. 5, no. 1, pp. 25-35, 2003.

[63] R. Kantor, D. A. Katzenstein, B. Efron et al., "Impact of HIV1 subtype and antiretroviral therapy on protease and reverse transcriptase genotype: results of a global collaboration," PLoS Medicine, vol. 2, Article ID e112, 2005.

[64] E. A. Soares, A. F. Santos, T. M. Sousa et al., "Differential drug resistance acquisition in HIV-1 of subtypes B and C," PloS ONE, vol. 2, no. 1, article e730, 2007.

[65] E. Sprinz, E. M. Netto, M. Patelli et al., "Primary antiretroviral drug resistance among HIV type 1-infected individuals in Brazil," AIDS Research and Human Retroviruses, vol. 25, no. 9, pp. 861-867, 2009.

[66] R. Kantor, R. W. Shafer, and D. Katzenstein, "The HIV-1 Non-subtype B workgroup: an international collaboration for the collection and analysis of HIV-1 non-subtype B data," MedGenMed, vol. 7, no. 1, article 71, 2005.

[67] G. S. Gottlieb, N. M. D. Badiane, S. E. Hawes et al., "Emergence of multiclass drug-resistance in HIV-2 in antiretroviral-treated individuals in Senegal: implications for HIV-2 treatment in resouce-limited West Africa," Clinical Infectious Diseases, vol. 48, no. 4, pp. 476-483, 2009.

[68] M. L. Ntemgwa, T. D. Toni, B. G. Brenner, R. J. Camacho, and M. A. Wainberg, "Antiretroviral drug resistance in human 
immunodeficiency virus type 2," Antimicrobial Agents and Chemotherapy, vol. 53, no. 9, pp. 3611-3619, 2009.

[69] M. Sylla, A. Chamberland, C. Boileau et al., "Characterization of drug resistance in antiretroviral-treated patients infected with HIV-1 CRF02_AG and AGK subtypes in Mali and Burkina Faso," Antiviral Therapy, vol. 13, no. 1, pp. 141-148, 2008.

[70] M. Kinomoto, R. Appiah-Opong, J. A. M. Brandful et al., "HIV-1 proteases from drug-naive West African patients are differentially less susceptible to protease inhibitors," Clinical Infectious Diseases, vol. 41, no. 2, pp. 243-251, 2005.

[71] H. J. Fleury, T. Toni, N. T. H. Lan et al., "Susceptibility to antiretroviral drugs of CRF01_AE, CRF02_AG, and subtype $C$ viruses from untreated patients of Africa and Asia: comparative genotypic and phenotypic data," AIDS Research and Human Retroviruses, vol. 22, no. 4, pp. 357-366, 2006.

[72] K. A. Delviks-Frankenberry, G. N. Nikolenko, F. Maldarelli, S. Hase, Y. Takebe, and V. K. Pathak, "Subtype-specific differences in the human immunodeficiency virus type 1 reverse transcriptase connection subdomain of CRF01-AE are associated with higher levels of resistance to $3^{\prime}$ azido- $3^{\prime}$ deoxythymidine," Journal of Virology, vol. 83, no. 17, pp. 8502$8513,2009$.

[73] K. A. Delviks-Frankenberry, G. N. Nikolenko, R. Barr, and V. K. Pathak, "Mutations in human immunodeficiency virus type 1 RNase H primer grip enhance 3 ' -azido-3' -deoxythymidine resistance," Journal of Virology, vol. 81, no. 13, pp. 6837-6845, 2007.

[74] S. H. Yap, C. W. Sheen, J. Fahey et al., "N348I in the connection domain of HIV-1 reverse transcriptase confers zidovudine and nevirapine resistance," PLoS Medicine, vol. 4, no. 12, Article ID e335, 2007.

[75] R. K. Gupta, A. Kohli, A. L. McCormick, G. J. Towers, D. Pillay, and C. M. Parry, "Full-length HIV-1 gag determines protease inhibitor susceptibility within in-vitro assays," AIDS, vol. 24, no. 11, pp. 1651-1655, 2010.

[76] A. F. Santos, D. M. Tebit, M. S. Lalonde et al., "The role of natural polymorphisms in HIV-1 CRF02_AG protease on protease inhibitor hypersusceptibility," Antimicrobial Agents and Chemotherapy, vol. 56, no. 5, pp. 2719-2725, 2012.

[77] T. Bar-Magen, D. A. Donahue, E. I. McDonough et al., "HIV1 subtype $\mathrm{B}$ and $\mathrm{C}$ integrase enzymes exhibit differential patterns of resistance to integrase inhibitors in biochemical assays," AIDS, vol. 24, no. 14, pp. 2171-2179, 2010.

[78] Y. Goldgur, R. Craigie, G. H. Cohen et al., "Structure of the HIV-1 integrase catalytic domain complexed with an inhibitor: a platform for antiviral drug design," Proceedings of the National Academy of Sciences of the United States of America, vol. 96, no. 23, pp. 13040-13043, 1999.

[79] T. Bar-Magen, R. D. Sloan, V. H. Faltenbacher et al., "Comparative biochemical analysis of HIV-1 subtype B and C integrase enzymes," Retrovirology, vol. 6, article 103, 2009.

[80] B. G. Brenner, M. Lowe, D. Moisi et al., "Subtype diversity associated with the development of HIV-1 resistance to integrase inhibitors," Journal of Medical Virology, vol. 83, no. 5, pp. 751-759, 2011.

[81] E. Z. Loizidou, I. Kousiappa, C. D. Zeinalipour-Yazdi, D. A. M. C. Van de Vijver, and L. G. Kostrikis, "Implications of HIV-1 M group polymorphisms on integrase inhibitor efficacy and resistance: genetic and structural in silico analyses," Biochemistry, vol. 48, no. 1, pp. 4-6, 2009.

[82] T. Bar-Magen, R. D. Sloan, D. A. Donahue et al., "Identification of novel mutations responsible for resistance to
MK-2048, a second-generation HIV-1 integrase inhibitor," Journal of Virology, vol. 84, no. 18, pp. 9210-9216, 2010.

[83] I. Malet, V. Fourati, C. Charpentier et al., "The HIV1 integrase G118R mutation confers raltegravir resistance to the CRF02_AG HIV-1 subtype," Journal of Antimicrobial Chemotherapy, vol. 66, pp. 2827-2830, 2011.

[84] K. E. Hightower, R. Wang, F. Deanda et al., "Dolutegravir (S/GSK1349572) exhibits significantly slower dissociation than raltegravir and elvitegravir from wild-type and integrase inhibitor-resistant HIV-1 integrase-DNA complexes," Antimicrobial Agents and Chemotherapy, vol. 55, pp. 4552-4559, 2011.

[85] P. K. Quashie, T. Mesplede, Y. S. Han et al., "Characterization of the R263K mutation in HIV-1 integrase that confers lowlevel resistance to the second-generation integrase strand transfer inhibitor dolutegravir," Journal of Virology, vol. 86, no. 5, pp. 2696-2705, 2012.

[86] A. U. Scherrer, B. Ledergerber, V. von Wyl et al., "Improved virological outcome in White patients infected with HIV-1 non-B subtypes compared to subtype B," Clinical Infectious Diseases, vol. 53, pp. 1143-1152, 2011.

[87] E. A. Soares, A. F. Santos, and M. A. Soares, "HIV-1 subtype and virological response to antiretroviral therapy: acquired drug resistance," Clinical Infectious Diseases, vol. 54, pp. 738$739,2012$.

[88] A. Velazquez-Campoy, S. Vega, and E. Freire, "Amplification of the effects of drug resistance mutations by background polymorphisms in HIV-1 protease from African subtypes," Biochemistry, vol. 41, no. 27, pp. 8613-8619, 2002.

[89] J. B. Nachega, M. Hislop, D. W. Dowdy, R. E. Chaisson, L. Regensberg, and G. Maartens, "Adherence to nonnucleoside reverse transcriptase inhibitor-based HIV therapy and virologic outcomes," Annals of Internal Medicine, vol. 146, no. 8, pp. 564-573, 2007.

[90] N. Richard, M. Juntilla, A. Abraha et al., "High prevalence of antiretroviral resistance in treated Ugandans infected with non-subtype B human immunodeficiency virus type 1," AIDS Research and Human Retroviruses, vol. 20, no. 4, pp. 355-364, 2004.

[91] H. T. Xu, J. L. Martinez-Cajas, M. L. Ntemgwa et al., "Effects of the K65R and K65R/M184V reverse transcriptase mutations in subtype C HIV on enzyme function and drug resistance," Retrovirology, vol. 6, article 14, 2009. 

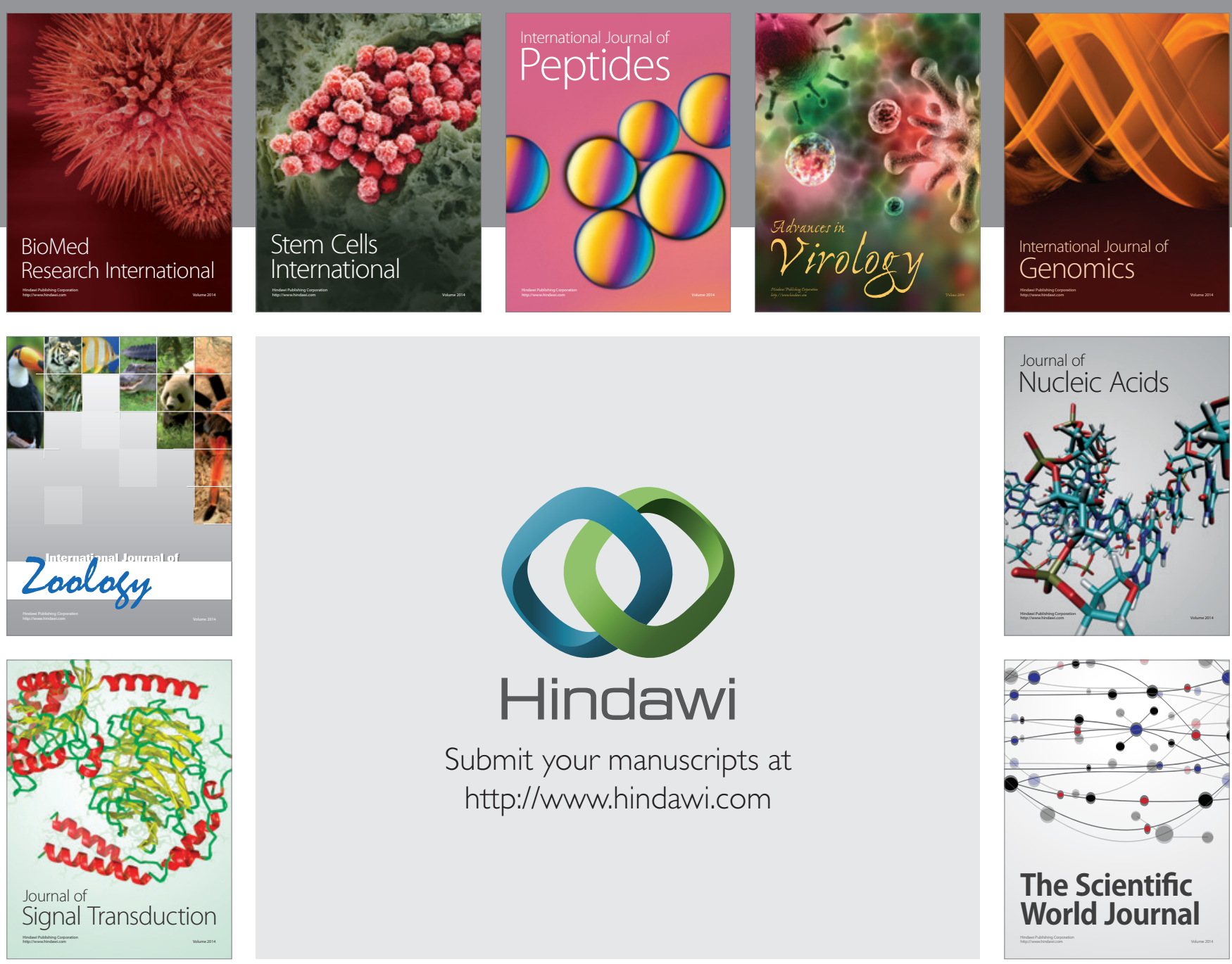

Submit your manuscripts at

http://www.hindawi.com
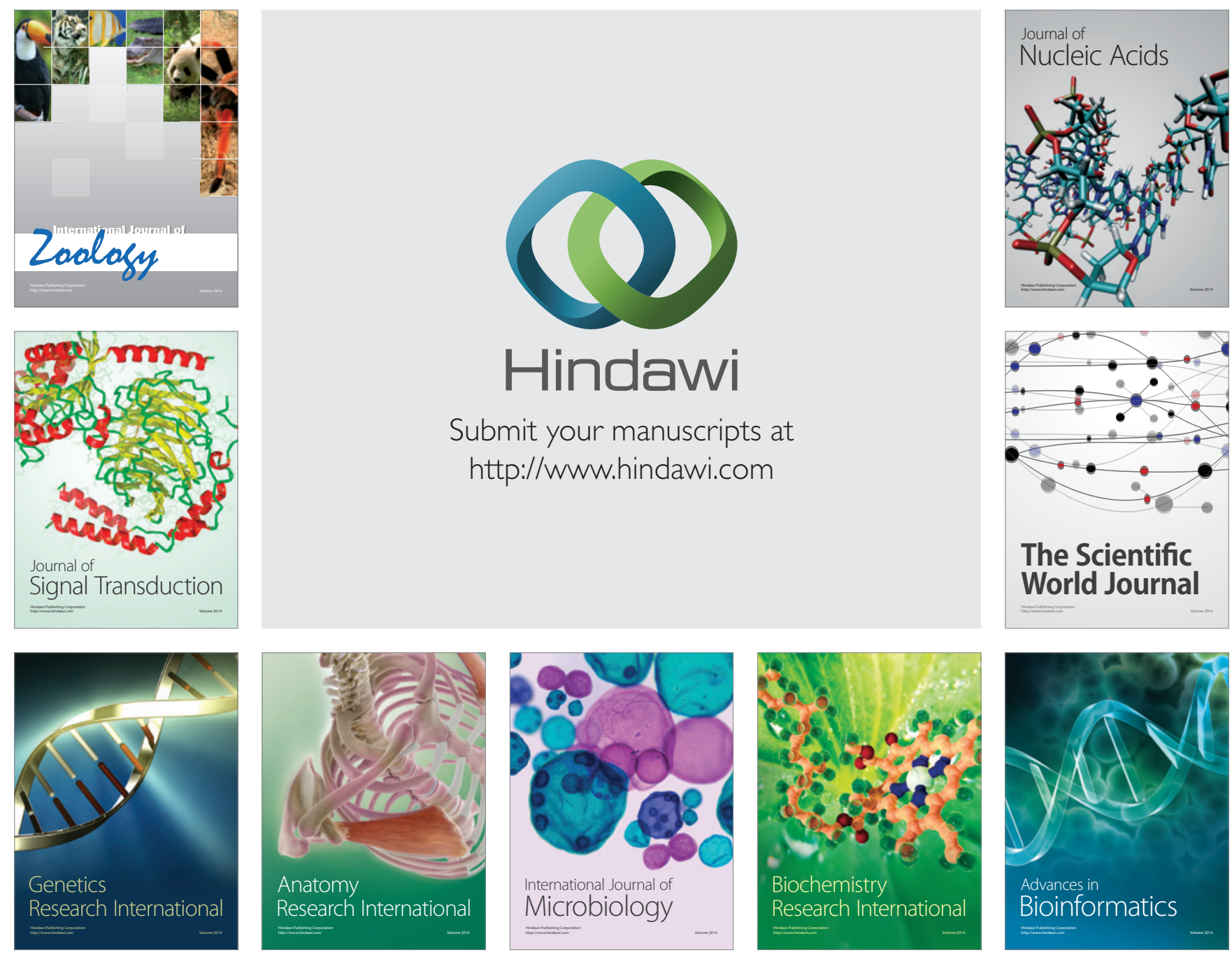

The Scientific World Journal
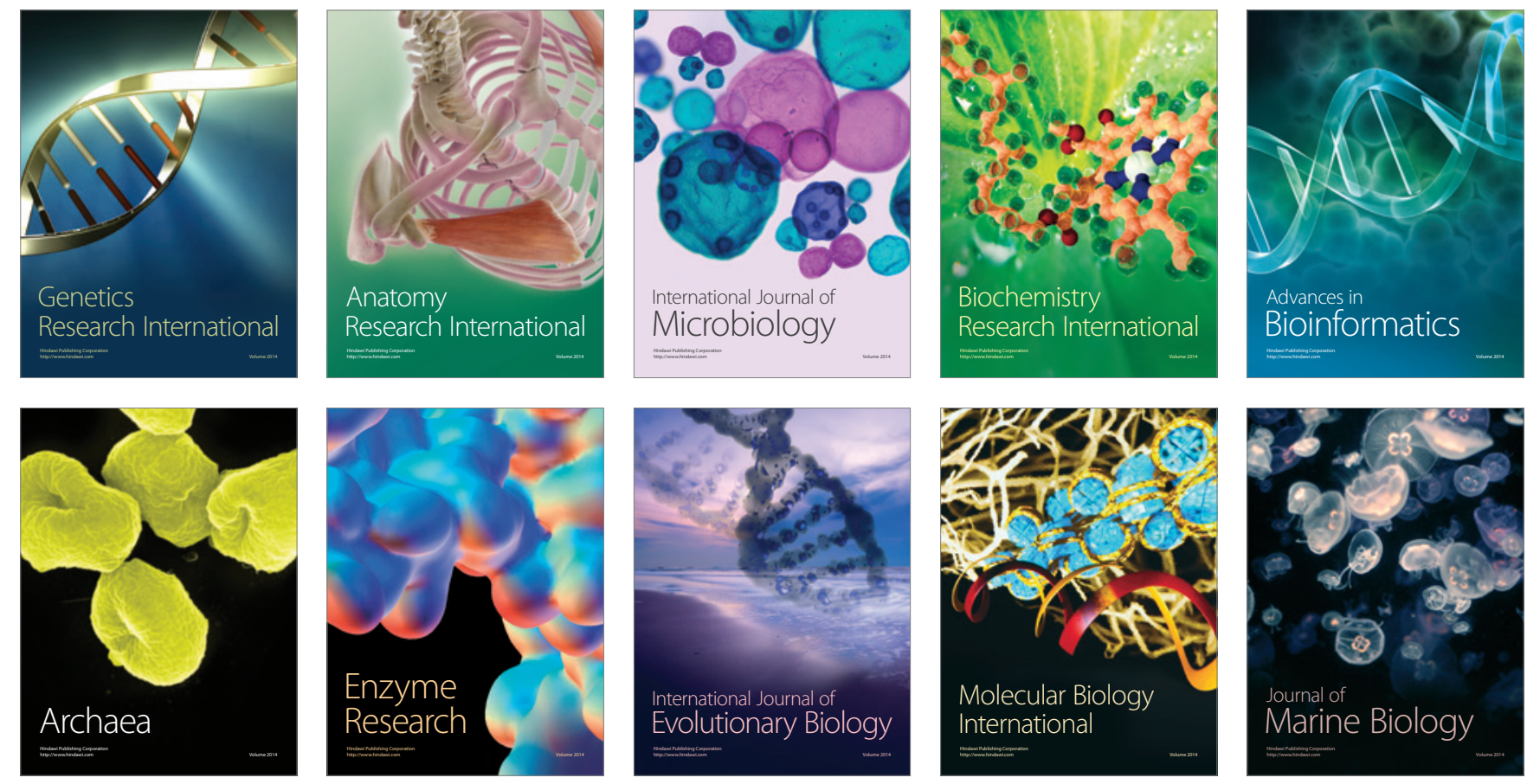